\title{
Comparative analysis of the oscillations in the diffraction cone
}

\author{
Jenö Kontros*, Kornél Kontros and Alexander Lengyel † \\ Institute of Electron Physics, Universitetska 21, 88016 Uzhgorod, Ukraine \\ E-mail: 'jeno@kontr.uzhgorod.ual, 'sasha@len.uzhgorod.ua'
}

\begin{abstract}
The oscillating structure in the $\bar{p} p-$ and $p p$-elastic scattering differential cross section at high energy and small momentum transfer is found by the maximumentropy method and the overlapping bins procedure. A comparative analysis of these two methods of identifying the oscillations was performed. We have concluded that the methods give qualitatively the same results.
\end{abstract}

\section{Introduction}

The oscillating structure of the diffraction peak in the differential $\bar{p} p-$ and $p p$-elastic cross

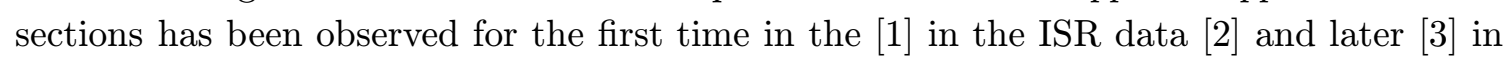
UA4/2 [i] experiment by normalizing the differential cross-section to the smoothly varying background in the impact parameter representation. In [5] an attempt was made to relate the observed structure near $|t|=0.1 \mathrm{GeV}^{2}$ to the variation of the opacity in $b$-space, probably reflecting the density oscillation in matter. The possible existence of oscillations with even smaller periods was discussed by several authors $\left[\begin{array}{l}\overline{6}_{1}^{\prime} \\ \underline{\underline{7}} \\ \underline{1}\end{array}\right]$.

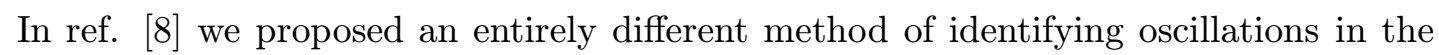
$\bar{p} p-$ and $p p$-elastic scattering. Our method was based on the use of overlapping bins of local slopes and we indicated some characteristic oscillation periods. It is quite obvious that, in order to determine the nature and periods of oscillations, one has first of all to increase the reliability of initial information contained in experimental data by suppressing the influence of statistical fluctuations. This problem can be settled by means of the well known method of maximum entropy [igi], used in many areas of physics. Recently it has been applied [i] $10 \overline{0} \mid \overline{1}]$ to the hadron scattering data.

\footnotetext{
${ }^{*}$ Speaker.

${ }^{\dagger}$ The authors are grateful to the Organizing Committee, in particular to Prof. A. Patkós for the possibility of participating to a nice Conference.
} 


\section{The maximum entropy method}

The mathematical essence of the maximum entropy method is formulated as follows. Provided there are $N$ measured data points $y_{k}$ with errors $\varepsilon_{k}$ and no theory available, the question arises how to choose the most plausible function $Y_{k}$ among all possible functions describing this data set. The philosophy is to choose the solution, which contains the least amount of information in order to avoid false features. Mathematically, this means that one has to choose such a function $Y_{k}$ that the functional $F$ :

$$
F=\chi^{2}+\lambda R
$$

is minimized. Here $\chi^{2}$ is the least-square term which provides the consistency of the function $Y$ with the data set $y, R$ is a regularization functional which helps to choose the correct function containing the least amount of information, and $\lambda$ is the weighting factor between the least-square term and the regularization functional. The value of $\lambda$ has to be chosen in such a way that the data are neither under- nor overestimated, i.e., $\chi^{2}$ should be equal to the number of data points $N$. The $\chi^{2}$ is calculated by means of the standard formula

$$
\chi^{2}=\sum_{k=1}^{N} \frac{\left(Y_{k}-y_{k}\right)^{2}}{\varepsilon_{k}^{2}} .
$$

It is very important to use realistic error values with method based on a regularization functional in order to obtain realistic results. In the maximum-entropy method the regularization functional is the configurational entropy of the distribution

$$
R=-\sum_{k=1}^{N} p_{k} \log \frac{p_{k}}{m_{k}}
$$

where

$$
p_{k}=\frac{Y_{k}}{\sum_{k=1}^{N} Y_{k}}
$$

with $m_{k}$ being the $k$-th value of the discretized a priori distribution. The basic principle of the maximum entropy method is that out of all probability distributions which satisfy given constraints, i.e. fits to the data, one should choose the distribution, which is the closest to the given a priori probability distribution $m_{k}$, and, if this is not specified, one should choose the distribution that is the closest to the uniform distribution. Here we should point out that unknown $Y_{k}$ are assumed to be independent of each other, which means that the distribution does not have to be continuous.

\section{Application of the maximum entropy method to the differential cross- section}

The question of the role of statistical errors in measured differential cross-section $\frac{d \sigma}{d t}(s, t)$ for $\bar{p} p$ - and $p p$-scattering at fixed $s$ within a broad $t$-interval is of substantial importance, 
because these errors for each data point may be of the same order of magnitude as the "fine" anomalies of $\frac{d \sigma}{d t}$ observed in experiments [2. 1. The second problem is related

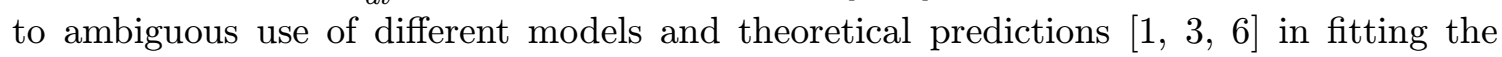
experimental "curves". From the standpoint of the "observed" oscillations, several methods are now being extensively used. One of the methods is based on theoretical description of smooth $\frac{d \sigma}{d t}$ and subsequent extraction of oscillations (anomalies) by subtracting the slowly varying "background" from the experimental differential cross sections. In our new approach, we first process the experimental $\left(\frac{d \sigma}{d t}\right)^{\text {exper }}$ values according to the "maximum entropy criterion" to obtain

$$
Y_{k}=\left(\frac{d \sigma}{d t}\right)_{k}^{e n t r}
$$

For this purpose we minimize the functional $(\overline{2} \overline{2} \cdot \overline{1})$, where

$$
y_{k}=\left(\frac{d \sigma}{d t}\right)_{k}^{\text {exper }}
$$

and $\varepsilon_{k}$ are experimental errors of $y_{k}$. We use the following parametrization

$$
\begin{gathered}
m_{k}=\left(\frac{d \sigma}{d t}\right)_{k}^{t h e o r}=\frac{1}{16 \pi}\left|F_{N}\right|^{2} \\
F_{N}=i \sigma_{t o t} \exp \left[b t_{k}\right]
\end{gathered}
$$

Here $\sigma_{\text {tot }}$ and $b$ are fitted param-

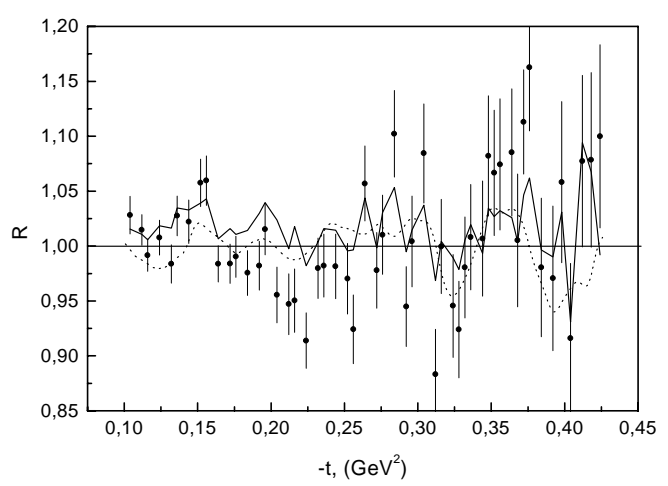

Figure 1: Experimental values of the data at $53 \mathrm{GeV}$ [2] were calculated by means of Eq. (3.5in) (points with error bars), maximum entropy fits with original errors (solid curve; $\chi^{2} / n d f \cong 1.01$ and $\lambda=0.23 \cdot 10^{6}$ ). Dotted curve was calculated by means of Eq. (5.2).

eters. We investigate the experimental differential $p p$-scattering cross sections at $\sqrt{s}=53$

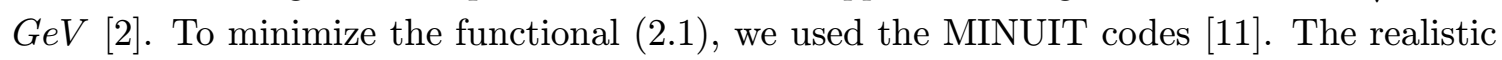
results were obtained when $\chi^{2} / n d f \cong 1$ was achieved. The results of the calculations with the a priori distribution described above are shown in Fig. 1 for the ratios

$$
\begin{gathered}
R_{k}^{\text {entr }}(t)=\frac{\left(\frac{d \sigma}{d t}\right)_{k}^{\text {entr }}}{\left(\frac{d \sigma}{d t}\right)_{k}^{\text {theor }}}, \\
R_{k}^{\text {exper }}(t)=\frac{\left(\frac{d \sigma}{d t}\right)_{k}^{\text {exper }}}{\left(\frac{d \sigma}{d t}\right)_{k}^{\text {theor }}},
\end{gathered}
$$

and the relevant errors. In spite of the decrease of the amplitude of oscillations, the local structure especially at $t \sim-0.1(\mathrm{GeV} / \mathrm{c})^{2}$ discussed in $\left.[\overline{\bar{p}}] \overline{0}\right]$ is still visible.

\section{The overlapping bins procedure for obtaining the local slope}

An entirely different method of identifying oscillations in the $\bar{p} p$ - and $p p$-elastic scattering was proposed in [i]. Our method was based on the use of overlapping bins of local slopes. 
To check the expected behaviour of the slope

$$
B(s, t)=\frac{d}{d t} \ln \left(\frac{d \sigma(s, t)}{d t}\right)
$$

over $t$ we shall operate with its "experimental" value.

Provided that

$$
\left(\frac{d \sigma}{d t}\right)=\left|a e^{b t}\right|^{2}
$$

has been measured for a given $s$ at $N|t|$-points lying in some interval $\left[|t|_{\min },|t|_{\max }\right]$, we adopt the following procedure. First, we divide this interval into subintervals or elementary "bins" (with $n_{b}$ measurements in each of them, assumed for simplicity to be the same for all bins). Once the first bin is chosen, the second bin is obtained from the first one by shifting only one point of measurement (of course, one could shift it by any number of points less or equal to $n_{b}$, the shift of one point is the minimal one giving rise to the maximal number of overlapping bins). The third bin is obtained from the second bin by shifting of one data point etc. Thus, we define $N-n_{b}+1$ overlapping bins for a given $s$. For each $(k$-th) bin, $n_{b}$ must be large enough and its width (in $\left.|t|\right)$ - small enough to allow fitting $\left(\frac{d \sigma}{d t}\right)$ with the simplest form directly involving the $t$-slope $b(4.2)$.

The parameter $b$ represents the value of the $t$-slope $B\left(<t>_{k}, s\right)$ "measured" at $s$ and "weighted average" $\langle t\rangle_{k}$ is defined in the $k$-th bin as

$$
<t>_{k}=\exp \left(\frac{\sum \frac{\left|t_{i}\right|}{\Delta y_{i}}}{\sum \frac{1}{\Delta y_{i}}}\right), \quad k \in\left[1, N-n_{b}+1\right]
$$

where $t_{i}$ is the value of $t$ at which the $\left(\frac{d \sigma}{d t}\right)_{i}$ is measured with the uncertainty $\Delta y_{i}$; the summations run over all data points, $i=1,2, \ldots, n_{b}$ of the bin. This yields the "experimental" values of $b_{k}\left(s, t_{k}\right)$ with the corresponding standard errors determined in the fit of (4) the data. Then the procedure is to be repeated for all bins and ultimately for the other $t$ 's at which the $\left(\frac{d \sigma}{d t}\right)$ have been measured. A regular structure in the slope of forward diffraction cone $B(s, t)$ is found (see Fig. 2).

\section{Fourier analysis of the results}

To elucidate the nature of the oscillations, we have subjected the obtained "experimental" data to a harmonic analysis. For this purpose, we write for the difference

$$
B(t)^{e x p}-B(t)^{\text {theor }}=\frac{a_{0}}{2}+\sum_{k=1}^{m}\left[a_{k} \cos \left(k \frac{2 \pi}{T} t\right)+b_{k} \sin \left(k \frac{2 \pi}{T} t\right)\right],
$$

where $m \leq n / 2$ and $n$ is the number of bins and $T$ denotes the $t$-interval considered. Usually, this interval spans from Coulomb interference region $\left(\sim 0.01 \mathrm{GeV}^{2}\right)$ up to the vicinity of the diffraction minimum $\left(\sim 0.8 \mathrm{GeV}^{2}\right)$. We have used a fairly large number of harmonics $(\sim 50)$ to account for the both large and small frequencies that may occur in slope parameter. The results are plotted in Fig. 2. The oscillations of the slope parameter 
are visible in the whole range considered. Then we calculated the ratio $R_{f}(t)$ using the obtained parameters from Fourier analysis

$$
R_{f}(t)=\exp \left\{\frac{a_{0} t}{2}+\sum_{k=1}^{m}\left[a_{k} \frac{T}{2 \pi k} \sin \left(k \frac{2 \pi}{T} t\right)-b_{k} \frac{T}{2 \pi k} \cos \left(k \frac{2 \pi}{T} t\right)\right]+c\right\}
$$

$c$ being an integration constant.

The resulting curve is shown in Fig. 1. Both curves have an irregular behaviour, coincide qualitatively with each other and follow well the typical shape of $R(t)$.

\section{Concluding remarks}

We conclude that in the analyzed cases the existing data on $p p$-elastic scattering reveal visible oscillation satisfying quite realistic reliability criteria. Both resulting curves (see Fig. 1) coincide qualitatively and show the same typical feature. After applying such procedures as the maximum entropy

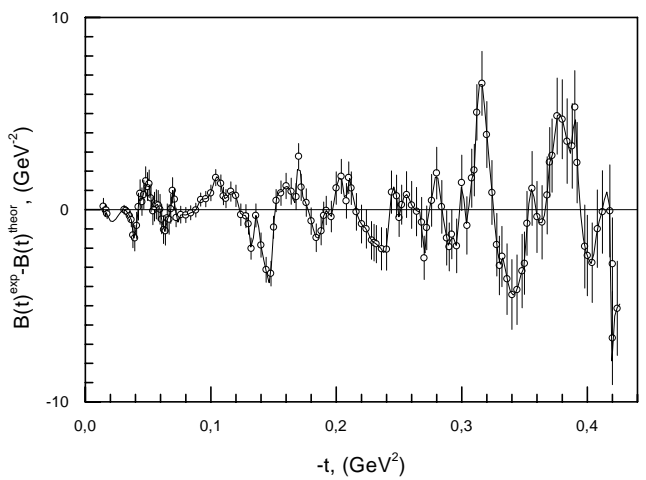

Figure 2: The oscillations of the slope parameter obtained by the overlapping bins method $B(t)^{\text {exp }}$ related to the smoothly varying function $B(t)^{t h e o r}$. The solid line was calculated with the help of Eq. (5.1).

and overlapping bins methods we can accumulate the data on the fine structure of the diffraction cone, and subsequently study in detail the nature of this phenomenon.

\section{References}

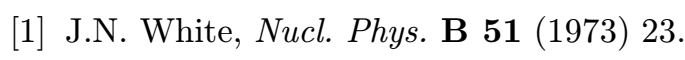

[2] B. Barbiellini et al, 2 h hys. Lett. B 390

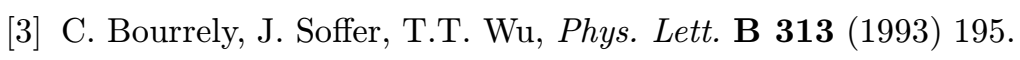

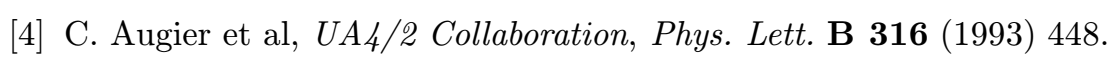

[5] A. Arnold, S. Barshay,

[6] S. Barshay, P. Heiliger,

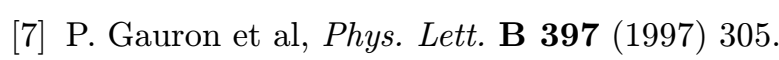

[8] J. Kontros, A. Lengyel, in Strong Interaction at Low Distances ed. L.L. Jenkovsky (Hadronic Press Palm Arbor) (1995) 67.

[9] Maximum Entropy in Action, (Oxford University Press) (1991).

[10] O. Dumbrajs, J. Kontros, A. Lengyel, UJ. Phys. $\mathbf{G} \mathbf{2} \mathbf{6}(20 \overline{0} 0) 132 \overline{1}$.

[11] CERN Computer Center Program Library, D506. 